\title{
Can hematological parameters in type 2 diabetes predict microvascular complication development?
}

\author{
Erhan Onalan', Nevzat Gozel², \\ Emir Donder ${ }^{3}$
}

\begin{abstract}
Objective: To examine potential associations between neutrophil/lymphocyte ratio, platelet/lymphocyte ratio, mean platelet volume (MPV), HbA1c and microvascular complications in diabetic patients from a cost-effectiveness perspective.

Methods: One hundred patients with type 2 diabetes attending our outpatient unit between May 2018 and October 2018 were included, and 100 healthy individuals served as the control group. A retrospective file search was performed to collect information on hemoglobin, mean platelet volume (MPV), glycosylated haemoglobin (HbA1c), hematocrit (Hct), neutrophil and lymphocyte count, neutrophil/lymphocyte ratio $(\mathrm{NLR})$, platelets (Plt), platelet/lymphocyte ratio (PLR), and microvascular complications (neuropathy, retinopathy, nephropathy).

Results: Demographic and laboratory data were retrospectively controlled between diabetes $(n=100)$ and healthy control $(n=100)$ groups. The mean age in diabetic patients and healthy controls was 56.34 and 36.68 years, respectively. The mean NLR in diabetics and healthy controls was 2.48 and 2.11, the difference in NLR being significant $(p=0.002)$. MPV in diabetics and controls was 8.54 and 8.53 , respectively, and the difference was not significant $(p=0.93)$. PLR was also similar, i.e. 149.7 and 145.3 in diabetics and healthy controls $(p=0.067)$. With respect to microvascular complications, retinopathy was found to be significantly associated with MPV and NLR $(p=0.015$, and $p=0.051)$, and nephropathy showed a significant association with NLR ( $p=0.027)$ among diabetics. In contrast with the two other microvascular complications, no significant association between neuropathy and NLR could be detected, while PLR and neuropathy was significantly associated $(\mathrm{p}=0.003)$.

Conclusion: Microvascular complications may be associated with certain hematologic parameters, as suggested by comparisons both between diabetics and healthy individuals and within the group of diabetic individuals. We believe that hematologic parameters such as hematocrit, MPV, NLR, and PLR, which can be obtained through a simple complete blood count, may be utilized as cost-effective predictors of diabetic microvascular complications. Further prospective studies with larger sample size are required to better delineate these associations.
\end{abstract}

KEYWORDS: Diabetes mellitus, Neutrophil/lymphocyte ratio, Platelet/lymphocyte ratio, Haemoglobin A1c.

doi: https://doi.org/10.12669/pjms.35.6.1150

How to cite this:

Onalan E, Gozel N, Donder E. Can hematological parameters in type 2 diabetes predict microvascular complication development? Pak J Med Sci. 2019;35(6):1511-1515. doi: https://doi.org/10.12669/pjms.35.6.1150

This is an Open Access article distributed under the terms of the Creative Commons Attribution License (http://creativecommons.org/licenses/by/3.0), which permits unrestricted use, distribution, and reproduction in any medium, provided the original work is properly cited.

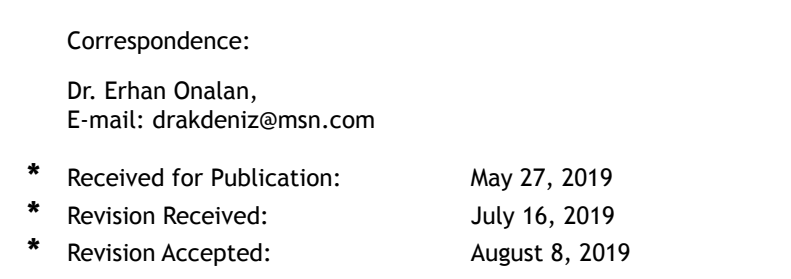

\section{INTRODUCTION}

Diabetes mellitus (DM) is a systemic and chronic metabolic disorder leading to chronic hyperglycemia that is characterized by disturbances by carbohydrate, protein and fat metabolism due to partial or complete lack of insulin and/or insulin 
resistance. Chronic hyperglycemia may not only lead to acute metabolic complications, but also long-term injury and dysfunction in several organs and systems of the body, particularly the eyes, kidneys, heart, and blood vessels. ${ }^{1}$

Neutrophil/lymphocyte ratio (NLR), platelet/ lymphocyte ratio (PLR), and platelet indices are inexpensive, practical, and easily accessible parameters readily estimated from complete blood count that have been found to be related with a number of medical conditions and pathologies. ${ }^{2-4}$ Studies reported correlations between these ratios and indices and metabolic/endocrinologic disorders. 5 Inflammatory processes play a key role in chronic diseases in many chronic conditions including cardiovascular disease, cancer, chronic kidney disease, and diabetes mellitus. ${ }^{7}$ Previous studies showed that the neutrophil/lymphocyte ratio (NLR) represents a systemic marker of inflammation, with a significant role in predicting the short-and long-term mortality for cardiovascular conditions and in predicting the prognosis in cancer patients. ${ }^{8,9}$

This study was undertaken to assess the utility of these simple, widely available, and inexpensive CBC parameters as markers of microvascular complications of diabetes and as predictors of potential complications.

\section{METHODS}

The study protocol was approved by the Ethics Committee approval of the Scientific Research Coordination Unit on 22.11.2018, with the reference number 08 at Firat University. Data were retrospectively collected for patients attending to the outpatient unit of General Internal Medicine, Medical Faculty of Firat University between May 2018 and October 2018. In addition to 100 study subjects with diabetes, 100 healthy individuals served as controls. Patients were included if they had no diagnosed conditions other than diabetes, were between 30-75 years of age, and were receiving oral anti-diabetics. Exclusion criteria were the presence of concomitant conditions (e.g. coronary artery disease, hematological conditions, malignancy, severe hepatic disorder, severe renal disorder), insulin use, and current smoking. A retrospective patient file search was performed to retrieve information on hemoglobin, mean platelet volume (MPV), glycosylated hemoglobin (HbA1c), hematocrit (Hct), neutrophil and lymphocyte count, neutrophil/lymphocyte ratio (NLR), platelets (Plt), platelet/lymphocyte ratio (PLR), and microvascular complications (neuropathy, retinopathy, nephropathy). Diabetic patients with $\mathrm{a}+$ proteinuria and/or a creatinine level $>1.2 \mathrm{mg} /$ $\mathrm{dl}$ were deemed to have diabetic nephropathy, and those diagnosed with diabetic retinopathy at the Department of Ophthalmology were deemed to have diabetic retinopathy. Patients with findings of polyneuropathy or neuropathy in EMG were considered to have diabetic neuropathy. The subset of patients with microvascular complicationts consisted of those with nephropathy, retinopathy, and/or neuropathy. Demographic data for the healthy controls and patients (i.e. age, gender, waist and hip circumference, body mass index) were collected from patient files.

Statistical Analyses: All statistical analyses were performed using Statistical Package for the Social Sciences (SPSS) 22.0 software pack. For data assessment, in addition to descriptive statistics [Mean $(\bar{X})$, Standard deviation (SD)], Student's $t$ test was used for the comparison of quantitative data with normal distribution, while one-way ANOVA was used for the comparisons between the groups. The significance of the difference between two statistically matched samples was tested with Wilcoxon's paired two sample test, while the chi-square test was used for the comparison of quantitative data. The results were expressed with $95 \%$ confidence intervals, and a $\mathrm{p}$ value of less than 0.05 was considered significant.

\section{RESULTS}

The comparison between diabetics and healthy controls with respect to demographic and laboratory parameters is shown in Table-I. The mean age in the diabetic group and healthy controls was 54.34 and 36.68 years, respectively. The mean NLR in diabetics and healthy controls was 2.48 and 2.11, the difference in NLR being significant $(p=0.002)$. MPV in diabetics and controls was 8.54 and 8.53, respectively, and the difference was not significant $(p=0.93)$. PLR was also similar, i.e. 149.7 and 145.3 in diabetics and healthy controls $(\mathrm{p}=0.067)$.

With respect to microvascular complications, retinopathy was found to be significantly associated with MPV and NLR $(p=0.015$, and $\mathrm{p}=0.051$ ), and nephropathy showed a significant association with NLR ( $p=0.027)$ among diabetics. In contrast with the two other microvascular 
Hematological parameters in type 2 diabetes

Table-I: Demographic and laboratory data in diabetic patients and healthy controls.

\begin{tabular}{|c|c|c|c|c|c|}
\hline & Groups & $N$ & Mean & Standard Deviation & p value \\
\hline \multirow[t]{2}{*}{ Gender (M/F) } & healthy & $46 / 54$ & & & \\
\hline & diabetic & $48 / 52$ & & & \\
\hline \multirow[t]{2}{*}{ Age (year) } & healthy & 100 & 36.68 & 10.756 & $<0.001$ \\
\hline & diabetic & 100 & 56.34 & 12.554 & \\
\hline \multirow[t]{2}{*}{ HbA1c $(\%)$} & healthy & 100 & 5.718 & 0.2938 & $<0.001$ \\
\hline & diabetic & 100 & 9.624 & 2.2356 & \\
\hline \multirow[t]{2}{*}{ Hematocrit (\%) } & healthy & 100 & 41.676 & 5.6697 & 0.736 \\
\hline & diabetic & 100 & 41.376 & 6.8421 & \\
\hline \multirow[t]{2}{*}{ Platelet $\left(x 10^{9} / \mathrm{L}\right)$} & healthy & 100 & 261620 & 55441.985 & 0.052 \\
\hline & diabetic & 100 & 279390 & 71906.771 & \\
\hline \multirow[t]{2}{*}{ Neutrophil $\left(x 10^{9} / \mathrm{L}\right)$} & healthy & 100 & 4081.00 & 1455.345 & 0.012 \\
\hline & diabetic & 100 & 4684.80 & 1888.585 & \\
\hline \multirow{2}{*}{ Lymphocyte (x109/L) } & healthy & 100 & 1972.40 & 452.028 & 0.044 \\
\hline & diabetic & 100 & 2201.70 & 1034.883 & \\
\hline \multirow[t]{2}{*}{ MPV (fl) } & healthy & 100 & 8.530 & 0.9422 & 0.930 \\
\hline & diabetic & 100 & 8.541 & 0.8186 & \\
\hline \multirow[t]{2}{*}{$\operatorname{NLR}(\%)$} & healthy & 100 & 2.1176 & 0.81013 & 0.022 \\
\hline & diabetic & 100 & 2.4823 & 1.35413 & \\
\hline \multirow[t]{2}{*}{ PLR (\%) } & healthy & 100 & 145.392 & 58.5490 & 0.677 \\
\hline & diabetic & 100 & 149.724 & 85.8579 & \\
\hline
\end{tabular}

NLR: Neutrophil/lymphocyte ratio; PLR: Platelet/lymphocyte ratio; MPV: Mean platelet volume.

complications, no significant association between neuropathy and NLR could be detected, while PLR and neuropathy was significantly associated $(\mathrm{p}=0.003)$ (Table-II).

\section{DISCUSSION}

Neutrophil/lymphocyte ratio (NLR), platelet/ lymphocyte ratio (PLR), and the platelet index

Table-II: The association of diabetic microvascular complications with MPV, NLR, and PLR.

\begin{tabular}{|c|c|c|c|c|c|}
\hline & Retinopathy & $N$ & Mean & Standard Deviation & Pvalue \\
\hline \multirow[t]{2}{*}{$\mathrm{MPV}(\mathrm{fl})$} & Present & 28 & 8,22 & 0,8562 & 0,015 \\
\hline & Absent & 72 & 8.66 & 0.7752 & \\
\hline \multirow{2}{*}{ NLR (\%) } & Present & 28 & 2.90 & 1.5572 & 0.051 \\
\hline & Absent & 72 & 2.31 & 1.2397 & \\
\hline \multirow[t]{2}{*}{ PLR (\%) } & Present & 28 & 146.8 & 64.254 & 0.834 \\
\hline & Absent & 72 & 150.8 & 93.295 & \\
\hline \multicolumn{6}{|c|}{ Nephropathy } \\
\hline \multirow[t]{2}{*}{$\operatorname{MPV}(\mathrm{fl})$} & Present & 22 & 8.30 & 0.791 & 0.118 \\
\hline & Absent & 78 & 8.60 & 0.818 & \\
\hline \multirow[t]{2}{*}{ NLR (\%) } & Present & 22 & 3.04 & 1.477 & 0.027 \\
\hline & Absent & 78 & 2.32 & 1.280 & \\
\hline \multirow[t]{2}{*}{ PLR (\%) } & Present & 22 & 149.3 & 55.390 & 0.982 \\
\hline & Absent & 78 & 149.8 & 75.374 & \\
\hline \multicolumn{6}{|c|}{ Neuropathy } \\
\hline \multirow[t]{2}{*}{$\operatorname{MPV}(\mathrm{fl})$} & Present & 74 & 8.47 & 0.784 & 0.198 \\
\hline & Absent & 26 & 8.71 & 0.902 & \\
\hline \multirow[t]{2}{*}{ NLR (\%) } & Present & 74 & 2.39 & 1.293 & 0.280 \\
\hline & Absent & 26 & 2.73 & 1.019 & \\
\hline \multirow[t]{2}{*}{ PLR (\%) } & Present & 74 & 134.7 & 56.90 & 0.003 \\
\hline & Absent & 26 & 192.4 & 131.05 & \\
\hline
\end{tabular}

NLR: Neutrophil/lymphocyte ratio; PLR: Platelet/lymphocyte ratio; MPV: Mean platelet volume. 
represent inexpensive, practical, and widely available laboratory parameters that can be readily estimated from routine complete blood counts. The association between these parameters and many medical conditions and pathologies has been clearly established..$^{2-4}$ Also, these indices and ratios have been reported to be associated with several metabolic and endocrinologic disorders. 5,6

In our study, there was a significant difference in terms of NLR between diabetics and healthy controls $(p=0.002)$. In many epidemiological studies, chronic inflammation has been reported to play a significant role in the pathogenesis of chronic diseases such as metabolic syndrome, hypertension, and diabetes. ${ }^{10,11}$ Cross-sectional and prospective studies also have indicated a positive correlation between Type 2 diabetes and its complications and C-reaktif protein(CRP), Interleukin-6(IL-6), and leukocyte counts. ${ }^{12}$

One study examining the association between gestational diabetes and mean platelet volume (MPV) showed significantly higher MPV values women with gestational diabetes as compared to those without this condition. Furthermore, MPV was reported to be correlated with the insulin resistance index, i.e. (Homeostatic Model Assessment for Insulin Resistance) HOMA-IR. ${ }^{13}$ While significant correlations between neuropathy and MPV were observed in some studies, in the study by Hekimsoy et al. involving 145 diabetic and 100 non-diabetic individuals, no statistically significant differences were foun. ${ }^{14,15}$ Similarly, we failed to detect differences in MPV between diabetics and healthy controls in our study. On the other hand, increased NLR values were found to represent a weak prognostic indicator in patients undergoing cardiovascular interventions. In some studies, elevated NLR was also found to be linked with increased mortality. ${ }^{14,15}$ For instance, a study examining the effect of cigarette smoking, several indices including NLR, PLR and the platelet index were higher among smokers, and NLR and platelet values were reported to be associated with the intensity of smoking. ${ }^{15}$ Therefore, such factors should be taken into consideration when assessing NLR, PLR, and other similar indices.

In the current study, a similar connection between NLR and the microvascular complications of diabetes such as retinopathy and nephropathy was observed, while no such correlations could be identified for neuropathy. Again, MPV and NLR were significantly associated with retinopathy and
NLR with nephropathy. In contrast with the two other microvascular complications, neuropathy did not significantly correlate with NLR, while PLR and nephropathy were significantly associated. The similarities and disagreements between our results and previous publications may be partially explained by the limitations of our study. Also, absence of a homogeneous distribution of the patients with regard to age, gender, oral antidiabetic use, and body mass index in this cross-sectional study represents one such limitation.

Limitations of the study: Our study should be evaluated in the light of several limitations. The presented study was conducted on a retrospective basis and represented single-center experience.

\section{CONCLUSION}

In conclusion, based on the comparisons between diabetic patients and healthy controls and within the group of diabetic individuals, our results seem to suggest that microvascular complications may be linked with certain hematological parameters in type 2 DM patients. Hematocrit, MPV, NLR, and PLR values have been found to be cost-effective parameters that predict diabetic microvascular complications and that can be readily estimated using a simple laboratory test such as the complete blood count. Further studies with larger sample size are warranted to better elucidate the agreement and inconsistency between our results and previous publications, and to reach firmer conclusions regarding the value of these indices.

Recommendations: Prospective and comprehensive studies that will include a larger number of patients are needed to confirm our findings.

Grant support, Financial \& Conflict of interest: None.

\section{REFERENCES}

1. American Diabetes Association. Diagnosis and classification of diabetes mellitus Diabetes Care. 2007;30:42-47. doi:10.2337/dc10-S062.

2. Tulgar YK, Cakar S, Tulgar S, Dalkilic O, Cakiroglu B, Uyanik BS. The effect of smoking on neutrophil/ lymphocyte and platelet/lymphocyte ratio and platelet indices: a retrospective study. Eur Rev Med Pharmacol. Sci. 2016;20:3112-3118.

3. Koh CH, Bhoo-Pathy N, Ng KL, Jabir RS, Tan GH, See $\mathrm{MH}$, et al. Utility of pre-treatment neutrophil-lymphocyte ratio and platelet-lymphocyte ratio as prognostic factors in breast cancer. Br J Cancer. 2015;113:150-158. doi: 10.1038/bjc.2015.183. 
4. Akdag S, Akyol A, Asker M, Duz R, Gumrukcuoglu HA. Platelet-to-Lymphocyte Ratio May Predict the Severity of Calcific Aortic Stenosis. Med Sci Monit. 2015;21:3395-3400. doi: 10.12659/MSM.894774.

5. Demirtas L, Degirmenci $\mathrm{H}$, Akbas EM, Ozcicek A, Timuroglu A, Gurel A, et al. Association of hematological indicies with diabetes, impaired glucose regulation and microvascular complications of diabetes. Int J Clin Exp Med. 2015;8:11420-11427.

6. Yilmaz H, Ucan B, Sayki M, Unsal I, Sahin M, Ozbek M, et al. Usefulness of the neutrophil-to-lymphocyte ratio to prediction of type 2 diabetes mellitus in morbid obesity. Diabetes Metab Syndr. 2015;9:299-304. doi: 10.1016/j. dsx.2014.04.009.

7. Manabe I: Chronic inflammation links cardiovascular, metabolic and renal diseases. Circ J. 2011;75:2739-2748. doi: 10.1253/circj.CJ-11-1184.

8. Azab B, Zaher M, Weiserbs KF, Torbey E, Lacossiere K, Gaddam S, et al. Usefulness of neutrophil to lymphocyte ratio in predicting short- and long-term mortality after non-ST-elevation myocardial infarction. Am J Cardiol. 2010;106:470-476. doi: 10.1016/j.amjcard.2014.10.008.

9. Chua W, Charles KA, Baracos VE, Clarke SJ. Neutrophil/ lymphocyte ratio predicts chemotherapy outcomes in patients with advanced colorectal cancer. $\mathrm{Br} \mathrm{J}$ Cancer. 2011;104:1288-1295. doi: 10.1038/bjc.2011.100.

10. Pitsavos C, Tampourlau M, Panagiotakos DB, Skoumas $Y$, Chrysohoou C, Nomikos T, et al. Association between lowgrade systemic inflammation and type 2 diabetes mellitus among men and women from the ATTICA Study. Rev Diabet Stud. 2007;4(2):98-104. doi: 10.1900/RDS.2007.4.98.

11. Bell DS, O'Keefe JH. White cell count, mortality, and metabolic syndrome in the Baltimore longitudinal study of aging. J Am Coll Cardiol. 2007;50(18):1810-1881.
12. Denghan A, Kardys I, de Maat MP, Uitterlinden AG, Sijbrands EJ, Bootsma $\mathrm{AH}$, et al. Genetic variation, C-reactive protein levels, and incidence of diabetes. Diabetes. 2007;56(3):872-878. doi: 10.2337/db06-0922.

13. Baldane S, Ipekci SH, Kebapcilar A. Relationship between insulin resistance and mean platelet volume in gestational diabetes mellitus. J Lab Phys. 2015;7:112-115. doi: 10.4103/0974-2727.163134.

14. Tamhane UU, Aneja S, Montgomery D, Rogers EK, Eagle KA, Gurm HS. Association between admission neutrophil to lymphocyte ratio and outcomes in patients with acute coronary syndrome. Am J Cardiol. 2008;102(6):653-657. doi: 10.1016/j.amjcard.2008.05.006.

15. Duffy BK, Gurm HS, Rajagopal V, Gupta R, Ellis SG, Bhatt DL. Usefulness of an elevated neutrophil to lymphocyte ratio in predicting long-term mortality after percutaneous coronary intervention. Am J Cardiol. 2006;97(7):993-996. doi: 10.1016/j.amjcard.2005.10.034.

\section{Authors Contribution:}

EO: Conceived, designed, did statistical analysis and manuscript writing.

EO \& NG: Did data collection and manuscript review.

ED: Did manuscript review and final approval.
Authors:

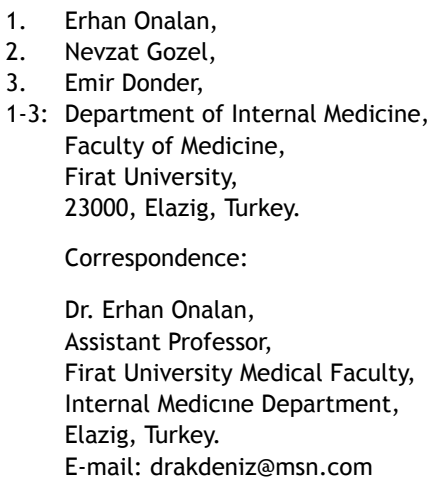

